Doi: HTTPS://DOI.ORG/10.23910/IJBSM/2017.8.4.1700

\title{
Field Evaluation of Bt. Cotton Hybrids Against Leaf Hoppers and Alternaria Leaf Spot
}

\author{
N. V. V. S. Durga Prasad, S. L. Bhattiprolu* and V. Chenga Reddy
}

Acharya N. G. Ranga Agricultural University, Regional Agricultural Research Station, Lam, Guntur, Andhra Pradesh (522 034), India

\section{Corresponding Author}

S. L. Bhattiprolu

e-mail: bhattiprolu2023@gmail.com

\author{
Article History \\ Article ID: AR1700 \\ Received in $29^{\text {th }}$ September, 2016 \\ Received in revised form $16^{\text {th }}$ July, 2017 \\ Accepted in $4^{\text {th }}$ August, 2017
}

\begin{abstract}
$B t$. cotton, transgenic to protect from boll worms, needs integrated pest/disease management to avoid the losses due to sucking pests and foliar diseases. It is important to identify Bt. cotton hybrids resistant to major pests and diseases. One hundred and forty three Bt. cotton hybrids were evaluated against leaf hopper incidence and Alternaria leaf spot diseases under unprotected conditions during $k$ harif, 2012. The experiment was laid in randomized block design, with two replications, by adopting $105 \times 60 \mathrm{~cm}^{2}$ spacing. Leaf hopper counts were taken at regular intervals and grading was done at peak incidence. Alternaria leaf spot disease was recorded by adopting 0 to 4 scales and percent disease intensity was calculated. Hybrid wise yield was recorded. Two hybrids viz., Tulasi-144 (Prachanda Bhaskar) BG-II (3.75PDI) and U5-SS-33 BG-II (4.38PDI) recorded resistant reaction while 127 hybrids were moderately resistant to Alternaria leaf spot. Fifteen hybrids viz., SWCH-4746 BG-II, Sri Tulasi BG-II-4, NCS 9605, SP 7149, Big boss, ACH-111-2, PCH 468, NCS-456, NCS-245, KSPL (KDCHH-065), NCS-9015 (Malini), Jodha, Lotus, Bunny and Badhra recorded moderate susceptibility. Thirty hybrids exhibited grade II reaction to leaf hoppers including Jadoo KCH-14K-59, Neeraja MRC-7201, ATM-KCH-311 BG-II, Yuva BG II, Dr. Brent MRC-7347 BG-II with moderate resistance to Alternaria leaf spot and yields above $20 \mathrm{q} \mathrm{ha}^{-1}$. In conclusion farmers are advised to cultivate $B t$. hybrids with multiple disease resistance to reduce the cost of plant protection and thus net returns can be enhanced.
\end{abstract}

Keywords: Cotton, leaf hopper, Alternaria leaf spot, resistance

\section{Introduction}

Cotton is an important commercial crop in India with a production of $35.2 \mathrm{~m}$ bales of $170 \mathrm{~kg}$ lint in 2015-2016 from an area of 11.88 mha with a productivity of $503 \mathrm{~kg} \mathrm{ha}^{-1}$, which is far behind the leading countries. Andhra Pradesh stood $3^{\text {rd }}$ in area ( $0.66 \mathrm{~m} \mathrm{ha}$ ) but $4^{\text {th }}$ in production ( $1.91 \mathrm{~m}$ bales) and $2^{\text {nd }}$ in productivity (641 kg ha-1) during 2015-2016 (Anonymous, 2016). The estimated loss due to sucking pests is up to $21.20 \%$ (Dhawan and Sidhu, 1986; Dhawan et al., 1988). Leaf hoppers are reported to cause $18.78 \%$ decline in cotton yield. Chavan et al. (2010) reported $28.13 \%$ avoidable yield loss due to major sucking pests in cotton. The extent of avoidable loss was $11.9 \%$ in MRC 6301 Bt. as against 11.2\% in RCH $134 \mathrm{Bt}$ and $16.2 \%$ in NCS 145 BG II (Shera, 2012) while Ramalakshmi et al. (2015) reported $13.13 \%$ losses in Tulasi-9 NBt, 19.99\% in RCH-2 NBt, $15.23 \%$ in Tulasi-9 BG-II and $16.82 \%$ yield losses in RCH-2 BG-II due to sucking pests. Leaf spot blight ${ }^{-1}$ caused by Alternaria macrospora Zimm. is the most commonly occurring disease in Andhra Pradesh causing losses to the tune of $38.23 \%$ in cotton variety LRA 5166 (Bhattiprolu and Rao, 2009) and 33.43\% in variety Jayadhar (Chattannavar et al., 2010).

The low productivity in Andhra Pradesh is attributed to cultivation of more than $60 \%$ of cotton under rainfed conditions by small and marginal farmers. Plant protection costs constitute considerable part of cost of cultivation. Developing low cost technologies will help to reduce the burden of poor tenant farmers. Management of pests and diseases through use of resistant varieties and hybrids constitutes an important strategy of integrated plant protection. Identification of resistant sources goes in this direction. A number of Bt. hybrids developed in India, to manage the bollworms, are being cultivated by farmers. However, these Bt. cotton hybrids need to be protected from sucking pests as well as important diseases. It is necessary to evaluate these hybrids to identify the resistant ones to major sucking pest, leaf hopper and major disease, Alternaria leaf spot. With this objective present studies were taken up.

\section{Materials and Methods}

A field experiment was conducted at Regional Agricultural Research Station, Lam, Guntur during kharif, 2012. One hundred and forty three $B t$. hybrids were sown with $105 \times 60$ $\mathrm{cm}^{2}$ spacing, in randomized block design with two replications. These hybrids were evaluated against leaf hopper incidence and Alternaria leaf spot diseases under unprotected 
conditions. Leafhopper counts were taken at regular intervals and grading was done at peak incidence.

Leafhopper grade: Grade 1: Normal leaf

Grade 2: Yellowing of leaves

Grade 3: Brick red leaf margins

Grade 4: Brick red leaves

Alternaria leaf spot disease was recorded by adopting 0 to 4 scales (Raj, 1988) (Table 1) and percent disease intensity was

Table 1: Scale Adopted for Alternaria leaf spot disease of cotton

\begin{tabular}{lll}
\hline Scale & Grade & \% leaf area infected \\
\hline 0 & Immune (I) & $\begin{array}{l}\text { Completely free from } \\
\text { disease } \\
\text { Up to } 5 \%\end{array}$ \\
2 & Resistant (R) & $6-20 \%$ \\
3 & $\begin{array}{l}\text { Moderately resistant } \\
\text { (MR) }\end{array}$ & $21-40 \%$ \\
4 & $\begin{array}{l}\text { Moderately susceptible } \\
\text { Susceptible (S) }\end{array}$ & $>40 \%$ \\
\hline
\end{tabular}

calculated as Percent Disease Intensity (PDI)=Sum of all disease ratings $\times 100 /$ total number of ratings $\times$ maximum disease grade.

Hybrid wise yield was recorded and hybrids were divided into five groups viz., hybrids yielding $<5 q$ ha $^{-1} ; 5-10 q$ ha $^{-1} ; 10-15$ q ha-1, $15-20$ q ha $^{-1}$ and $>20$ q ha $^{-1}$.

\section{Results and Discussion}

Thirty hybrids exhibited grade II reaction to leaf hoppers including Avathar Super-BG-II, Jadoo KCH-14K-59, NSPL-252 BG-II, Chaitanya MRC-7377 BG-II, JKCH-99 BG-II, Neeraja MRC7201 BG-II, Bindas BG-II, Robo-SSCH-555 BG-II, ATM-KCH-311 BG-II, Dr. Brent MRC-7347 BG-II, PCH-1411, SP 7157, NCS-863, Robo-ZCH -541, PRCH-709, Ankur 3224, NCS-1818, PCH-4599, NCS-7788, PCH-888, Big boss, ACH-111-2, Guru-721 BG-II, PCH 468, NCS-456, NCS-9015 (Malini), SWCH-4765, Ajeeth-155, Badhra and Yuva while 93 hybrids responded with grade III reaction and twenty hybrids with grade IV reaction (Table 2 ).

According to Prasad and Rao (2008) both Bt. and non Bt. versions of Mallika, Proagro368 and Bunny hybrid were found moderately resistant against leaf hoppers with low seasonal mean incidence among the hybrids., while $\mathrm{RCH} 2 \mathrm{Bt}$. , $\mathrm{RCH} 2$ non $B t$. and $\mathrm{RCH} 20 \mathrm{Bt}$. were found more prone to leafhoppers. Ankur 3070 BG II, Durga BG, Atal BG II, Krish BG II, Ryan BG,

Table 2: Reaction of Bt. Cotton Hybrids to Leaf hopper

$\begin{array}{ll}\text { Leaf hopper } & \text { Hybrids } \\ \text { grade } & \end{array}$

I Nil

II (30) Avathar Super-BG-II, Jadoo KCH-14K-59, NSPL-252 BG-II, Chaitanya MRC-7377 BG-II, JKCH-99 BG-II, Neeraja MRC-7201 BG-II, Bindas BG-II, Robo-SSCH-555 BG-II, ATM-KCH-311 BG-II, Dr. Brent MRC-7347 BG-II, PCH1411, SP 7157, NCS-863, Robo-ZCH-541, PRCH-709, Ankur 3224, NCS-1818, PCH 4599, NCS-7788, PCH 888, Big boss, ACH-111-2, Guru-721 BG-II, PCH 468, NCS-456, NCS-9015 (Malini), SWCH-4765, Ajeeth-155, Badhra, Yuva

III (93) SWCH-4746 BG-II, President Gold ZCH-503, Ankur 3028 BG-II, Chiranjeevi BG-II, Pradhan (GK235), NCS-866 (Chirutha), ACH-177-2 (Arya), Jackpot KCH-15K-39, Sigma-555, RCH-659 BG-II, GK-228 BG-II, Trust K4, Brahma, Tulasi-118 (Taakath BG-II), NCS-860 (Utham), Chetak BG-II, JK Indra Vajra BG-II, Hanuman SSCH-333 BG-II, MRC-7351 BG-II, ACH-11-2, Sarpanch (GK 231), Tulasi-144 (Prachanda Bhaskar) BG-II, Polaris ZCH508, Megha BG-II Super-544, Sudarshan, Dyna K4, NCS-9014 (Sampada), RCH-111 BG-II, Vishwanath (NCEH 2R), Tulasi-216 (Sourav-II), PCH-731 (Srilatha), PRCH-739 (Komal), Chitra (NCEH-15), Krish SWCH-4708 BG-II, Gabbar BG-II, RCH-533 BG-II, PRCH-505 (Jumbo), RCH-665, Tulasi-9 (Bhaskar BG-II), JKCH-2245 BG-II, Sri Tulasi BG-II-4, Barood-KCH-36, Ankur 3034 BG II, Solar-75 BG-II, Solar-66 BG-II, JKCH-8836 BG-II, LEO-72 BGII, VICH-303 Bt. BG-II, U5-SS-33 BG-II, SP 1171, NCS 9028, NCS-950, PCH-9620 (Indraja), NCS-1111 (Sona), RCH-668 BG-II, Kuber (GBCH-85), NCS-927, VICH-301 Bt BG-II, PRCH-746, NCS-9030 (Vallabha), ACH-199-2, RCH-530 BG-II, NCS 9605, SP 7149, PRCH-703 (Denim), Rith - Super 511, Champion ZCH-504, NCS-909, PRCH745, Ankur 3244, Namcot 605, Pratheek (KDCHH-9632), Ankur 3228, NCS-864, Gayathri Gold 69.SS.66 BG-II, Solar-76 BG-II, Pancham (KDCHH-541), JKCH-8665, Dhruv Gold ZCH-501 BG-II, Diamond 54.SS.33 BG-II, GK221 BG-II, NCS-245, KSPL (KDCHH-065), PRCH-710 (Odin), NCS-1134 (Suraksha), RCH-20 BG-II, Jodha, Lotus, Surya (Tulasi-171), Mallika, Mahathma, Bunny, Mallika Gold.

IV (20) RCH-578 BG-II, Commando, Power BG-II, Sarju BG-II, Jagannath (NCEH-34), Solar-60 BG-II, Tulasi-252 (Vahini BG-II), Drona Gold, Jai BG-II, RCH-2, VICH-5 Bt. BG-II, Bliss, GK-218 BG-II, Namcot 615, Victor (10122), Namcot 614, Vardan (GBCH-95), Mahasangram BG-II, Namcot 612, Ankur 5642. 
Madhura BG, MRC 7301 BG II, SP 504 BG II, Namskar BG II, Mahi BG II, VICH 312 BG II, Ankur 3028 BG, Anvitha BG, VICH 304 BG II, Ankur 3034 BG II, VICH 311 BG II, Ankur Jai BG II, Ankur 216 BG II, Ankur 3042 BG II, Vanaja BG, VICH 301 BG II, Classic BG II, Bunny BG II, Menaka BG II, VICH 314 BG II, Veda 2 BG II, VICH 303 BG II were tolerant to leafhoppers (Nagrare et al., 2014). Jassid population was significantly higher on variety FH Bt.1000, while the insect population was slightly lower on check variety NIAB-78 and on variety $B t .886$ and the lowest on variety Bt.3701 (Solangi et al., 2014). Significantly highest $(p<0.01)$ population of jassid was recorded on Sindh1 followed by Bt. cotton, Shahbaz, Niab-78 and Hari dost (Lanjar et al., 2014). Cotton cultivar, FH-158 showed resistance against jassid while $\mathrm{FH}-172$ was resistant to both jassids and thrips (Shahid et al., 2015). Three Bt. cotton hybrids viz., Dr Brent BG II, Ajeet 155 and Rakhi BG II recorded lowest number of leaf hoppers, $0.51,0.53$ and 0.73 respectively while $37 \mathrm{Bt}$. hybrids showed mean incidence of 1.85 (Reddy et al., 2015). Six Bt. hybrids viz., Ajeet 155, Indra Vajra, Jackpot BG II, Mallika BG II, Sandeep and Pratheek BG II were resistant to both sucking pests and foliar diseases (Reddy et al., 2015).

None of the entries was immune to Alternaria leaf spot. Reaction of the entries is presented in Table 3.

Intensity of Alternaria leaf spot ranged from 3.75 to $35.63 \%$

Table 3: Reaction of Bt. cotton hybrids to Alternaria leaf spot

Grade Alternaria leaf spot

Immune Nil

Resistant (2) Tulasi-144 (Prachanda Bhaskar) BG-II and U5-SS-33 BG-II

Moderately President Gold ZCH-503, Avathar Super-BG-II, Ankur 3028 BG-II, Chiranjeevi BG-II, Pradhan (GK235), resistant (126) RCH-578 BG-II, Jadoo KCH-14K-59, Commando, NCS-866 (Chirutha), NSPL-252 BG-II, Chaitanya MRC7377 BG-II, ACH-177-2 (Arya), Power BG-II, Jackpot KCH-15K-39, Sigma-555, Sarju BG-II, Jagannath (NCEH-34), RCH-659 BG-II, GK-228 BG-II, Trust K4, Solar-60 BG-II, Tulasi-252 (Vahini BG-II), Brahma, JKCH99 BG-II, Tulasi-118 (Taakath BG-II), Neeraja MRC-7201 BG-II, Drona Gold, Jai BG-II, NCS-860 (Utham), Chetak BG-II, Bindas BG-II, JK Indra Vajra BG-II, Hanuman SSCH-333 BG-II, MRC-7351 BG-II, RCH-2, ACH11-2, Robo-SSCH-555 BG-II, Sarpanch (GK 231), Polaris ZCH-508, Megha BG-II Super-544, Sudarshan, Dyna K4, ATM- KCH-311 BG-II, VICH-5 Bt. BG-II, Bliss, NCS-9014 (Sampada), RCH-111 BG-II, Vishwanath (NCEH 2R), Tulasi-216 (Sourav-II), PCH-731 (Srilatha), PRCH-739 (Komal), Chitra (NCEH-15), Krish SWCH4708 BG-II, Gabbar BG-II, GK-218 BG-II, RCH-533 BG-II, PRCH-505 (Jumbo), RCH-665, Tulasi-9 (Bhaskar) BG-II, Dr. Brent MRC-7347 BG-II, JKCH-2245 BG-II, Barood-KCH-36, Ankur 3034 BG II, Solar-75 BG-II, Solar-66 BG-II, JKCH-8836 BG-II, LEO-72 BG-II, VICH-303 Bt. BG-II, PCH-1411, SP 1171, Namcot 615, NCS 9028,Victor (10122), SP 7157, NCS-863, NCS-950, PCH-9620 (Indraja), NCS-1111 (Sona), RCH-668 BGII, Robo-ZCH-541, Namcot 614, PRCH-709, Kuber (GBCH-85), NCS-927, VICH-301 Bt.BG-II, PRCH-746, NCS-9030 (Vallabha), ACH-199-2, RCH-530 BG-II, Ankur 3224, Vardan (GBCH-95), PRCH-703 (Denim), NCS-1818, Rith-Super 511, Champion ZCH-504, Mahasangram BG-II, NCS - 909, PCH 4599, NCS - 7788, PRCH - 745, Ankur 3244, Namcot 605, Pratheek (KDCHH-9632), Ankur 3228, PCH 888, NCS-864, Gayathri Gold 69.SS.66 BG-II, Guru-721 BG-II, Solar-76 BG-II, Namcot 612, Ankur 5642, Pancham (KDCHH-541), JKCH-8665, Dhruv Gold ZCH-501 BG-II, Diamond 54.SS.33 BG-II, GK-221 BG-II, PRCH-710 (Odin), NCS1134 (Suraksha), RCH-20 BG-II, Surya (Tulasi-171), Mallika, SWCH-4765, Ajeeth-155, Mahathma, Mallika Gold, Yuva

Moderately SWCH-4746 BG-II, Sri Tulasi BG-II-4, NCS 9605, SP 7149, Big boss, ACH-111-2, PCH 468, NCS-456, NCSsusceptible (15) 245, KSPL (KDCHH-065), NCS-9015 (Malini), Jodha, Lotus, Bunny, Badhra

Susceptible

among different hybrids. Two hybrids viz., Tulasi-144 (Prachanda Bhaskar) BG-II (3.75\%) and U5-SS-33 BG-II (4.38\%) recorded resistant reaction while 127 hybrids were moderately resistant to Alternaria leaf spot. Bhattiprolu and Prasad (2011) reported resistant nature of MRC-7201 BG II to Alternaria leaf spot while Mallika Gold Bt. 2, Mallika Bt. 2 and Mallika Bt. were moderately resistant. Bt. cotton hybrid, Ganesh BG II was resistant and $21 \mathrm{Bt}$. cotton hybrids showed moderately resistant reaction against Alternaria leaf spot (Reddy et al., 2015). Fifteen hybrids viz., SWCH-4746 BG-II, Sri Tulasi BG-II-4,
NCS 9605, SP 7149, Big boss, ACH-111-2, PCH 468, NCS-456, NCS-245, KSPL (KDCHH-065), NCS-9015 (Malini), Jodha, Lotus, Bunny and Badhra recorded moderate susceptibility.

Yield data showed that three Bt. cotton hybrids viz., ATM- $\mathrm{KCH}$ 311 BG-II, PRCH-709 and Guru-721 BG-II produced more than $30 \mathrm{q} \mathrm{ha}^{-1}$ (Table 4).

Eight hybrids viz., Bindas, Dr. Brent MRC-7347, NCS-863, Robo-ZCH -541, NCS-927, PRCH-746, PCH 4599 and Yuva yielded between 25 to $30 \mathrm{q} \mathrm{ha}^{-1}$ while twenty four $B t$. cotton 


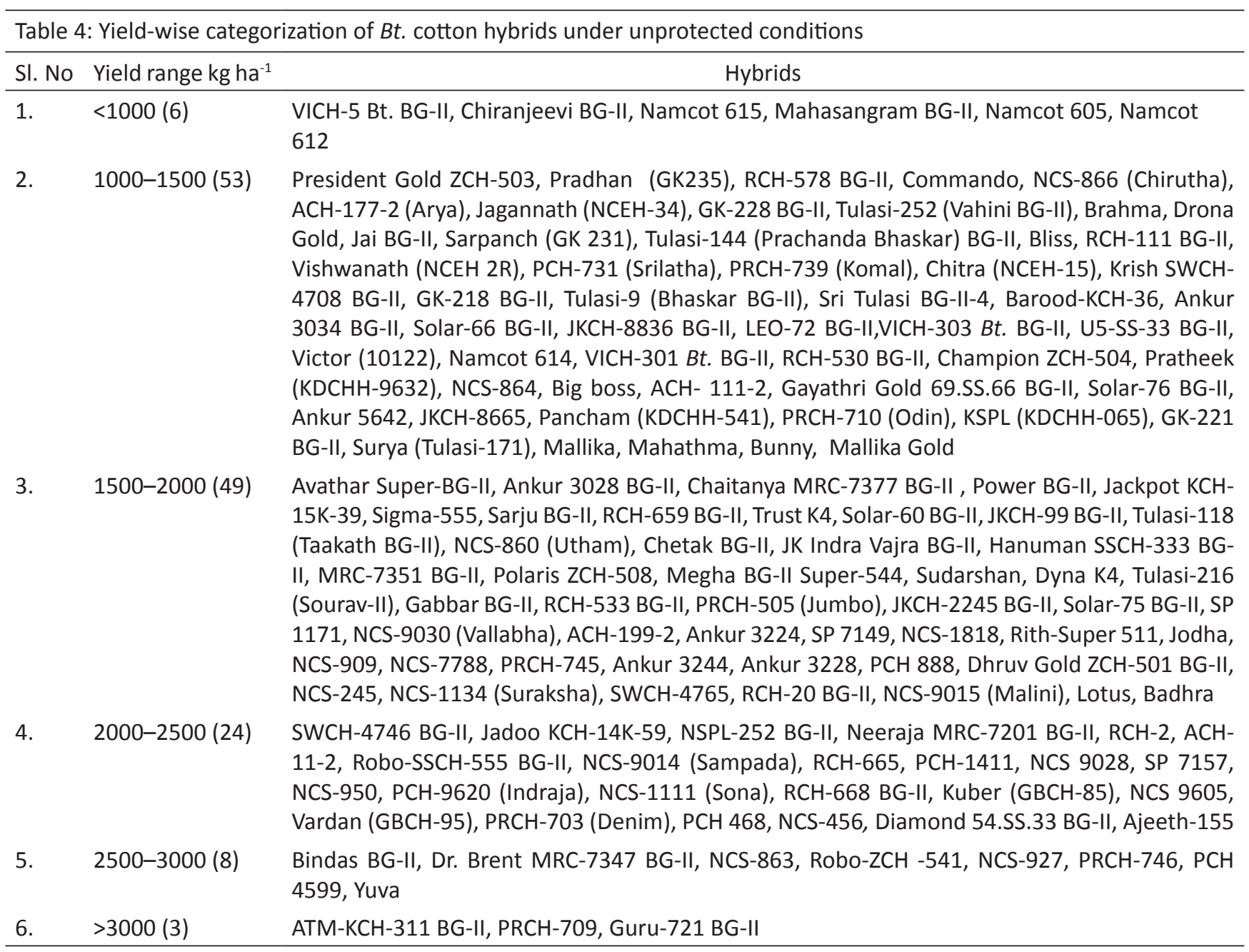

hybrids gave 20 to $25 \mathrm{q} \mathrm{ha}^{-1}$. It was observed that 30 hybrids were moderately resistant to Alternaria leaf spot, with grade II reaction to leaf hoppers and gave yields above $20 \mathrm{q} \mathrm{ha}^{-1}$. NCS 927 and PRCH 746 yielded 25-30 q ha ${ }^{-1}$ despite grade III reaction to leaf hoppers. Nagrare et al. (2014) recorded highest yields in the range of 1955 to $1539 \mathrm{~kg} \mathrm{ha}^{-1}$ in MRC $7301 \mathrm{BG}$ II, Krish BG II, RCH 530 BG II, Atal BG II, Bunny BG, Uttam BG II, Classic BG II, Shrimanth BG, ALTO BG II, Krishna BG II and Ryan $B G$ under rainfed farming. Four $B t$. cotton hybrids viz., Ajit 155, Dr Brent BG II, Neeraja BG II and SWCH 4769 BG II recorded more than $20 \mathrm{q} \mathrm{ha}^{-1}$, besides tolerance to the sucking pests and foliar diseases (Reddy et al., 2015).

\section{Conclusion}

Farmers, in particular, small and marginal as well as tenant farmers are advised to choose high yielding Bt. cotton hybrids with resistance to major sucking pest, leaf hoppers and disease, Alternaria leaf spot viz., ATM-KCH-311, PRCH-709, Guru-721, Bindas, Dr. Brent MRC-7347, NCS-863, Robo-ZCH -541, NCS-927, PRCH-746, PCH 4599 and Yuva which are proven promising under unprotected conditions. To maximize their net returns these hybrids can be grown with minimal plant protection for still better yields by minimizing the indiscriminate pesticide sprays.

\section{References}

Anonymous, 2016. Annual Report. All India Coordinated Cotton Improvement Project, Coimbatore.

Bhattiprolu, S.L., Rao, M.P.P., 2009. Estimation of crop losses due to Alternaria leaf spot in cotton. Journal of the Indian Society for Cotton Improvement 34(3), 151-154.

Bhattiprolu, S.L., Prasad, N.V.V.S.D., 2011. Field evaluation of $B t$. cotton hybrids against major diseases. Journal of Cotton Research and Development 25(1), 112-114.

Chattannavar, S.N., Hosagoudar, G.N., Ashtaputre, S.A., 2010. Crop loss estimation due to foliar diseases in cotton. Karnataka Journal of Agricultural Sciences 23(4), 602-605.

Chavan, S.J., Bhosle, B.B., Bhute, N.K., 2010. Estimation of losses due to major insect-pests in desi cotton in Maharashtra. Journal of Cotton Research and Development 24(1), 95-96.

Dhawan, A.K., Sidhu, A.S., 1986. Assessment of losses due to attack of cotton jassid on hirsutum cotton. Indian Journal 
of Plant Protection 14(1), 45-50.

Dhawan, A.K., Sidhu, A.S., Simwat, G.S., 1988. Assessment of avoidable loss in cotton (Gossypium hirsutum and G. arboreum) due to sucking pests and bollworms. Indian Journal of Agricultural Sciences 58(4), 290-292.

Lanjar, A.G., Solangi, B.K., Khuhro, S.A., Solangi, A.W., 2014. Insect infestation on Bt. and Non-Bt. Cotton cultivars. Food Science and Quality Management 27, 55-62. Available from http://www.iiste.org.

Nagrare, V.S., Deshmukh, A.J., Bisane, A.K., 2014. Relative performance of $B t$. cotton hybrids against sucking pests and leaf reddening under rainfed farming. Entomology, Ornithology \& Herpetology: Current Research 3(3), 134. Available from: http://dx.doi.org/10.4172/21610983.1000134

Prasad, N.V.V.S.D., Rao, N.H., 2008. Field evaluation of Bt. cotton hybrids against insect pest complex under rainfed conditions. Indian Journal of Entomology 70(4), 330-336.

Raj, S., 1988. Grading system for cotton diseases. Central Insitute for Cotton Research, Nagpur. Technical Bulletin, $1-7$.
Ramalakshmi, V., Rao, G.M.V.P., Madhumathi, T., 2015. Estimation of yield losses due to sucking pests in transgenic cotton Indian Journal of Plant Protection 43(1), 98-100.

Reddy, K.G., Bhattiprolu, S.L., Reddy, M.C.S., Reddy, V.C., 2015. Field evaluation of $B t$. cotton hybrids against certain sucking pests and foliar diseases. Journal of Research ANGRAU 43(3\&4), 42-47.

Shahid, M.R., Farooq, J., Mahmood, A., Iqbal, M.S., Mahmood, K., Abbas, H.G., 2015. Economic yield, fiber trait and sucking insect pest incidence on advanced genotypes of cotton in Pakistan Cercetari Agronomicein Moldova Vol. XLVIII, No. 1(161), 51-56.

Shera, P.S., 2012. Assessment of avoidable yield losses due to sucking pests in Bt. cotton hybrids. Journal of Cotton Research and Development 26(2), 254-257.

Solangi, B.K., Suthar, V., Sultana, R., Abassi, A.R., Hullio , N., Solangi, M.N., 2014. Relative resistance of cross Bt. cotton varieties against sucking complex, European Academic Research 2(6), 8434-8453. 